\title{
SCIENTIFIC REPORTS

\section{Age-related decrease in collagen proton fraction in tibial tendons estimated by magnetization transfer modeling of ultrashort echo time magnetic resonance imaging (UTE-MRI)}

Saeed Jerban $\mathbb{1}^{1 *}$, Yajun Ma ${ }^{1}$, Behnam Namiranian ${ }^{1}$, Aria Ashir ${ }^{1}$, Hoda Shirazian ${ }^{1}$, Zhao Wei $^{1}$, Nicole Le ${ }^{2}$, Mei Wu ${ }^{1}$, Zhenyu Cai ${ }^{1}$, Jiang Du ${ }^{1}$ \& EricY. Chang ${ }^{1,2^{*}}$

Clinical magnetic resonance imaging (MRI) sequences are not often capable of directly visualizing tendons. Ultrashort echo time (UTE) MRI can acquire high signal from tendons thus enabling quantitative assessments. Magnetization transfer (MT) modeling combined with UTE-MRI-UTE-MTmodeling-can indirectly assess macromolecular protons in the tendon. This study aimed to determine if UTE-MT-modeling is a quantitative technique sensitive to the age-related changes of tendons. The legs of 26 young healthy ( $29 \pm 6$ years old) and 22 elderly $(75 \pm 8$ years old) female subjects were imaged using UTE sequences on a 3T MRI scanner. Institutional review board approval was obtained, and all recruited subjects provided written informed consent. T1 and UTE-MT-modeling were performed on anterior tibialis tendons (ATT) and posterior tibialis tendons (PTT) as two representative human leg tendons. A series of $\mathrm{MT}$ pulse saturation powers $\left(500-1500^{\circ}\right)$ and frequency offsets $(2-50 \mathrm{kHz})$ were used to measure the macromolecular fraction (MMF) and macromolecular $\mathrm{T} 2\left(\mathrm{~T}_{\mathrm{MM}}\right)$. All measurements were repeated by three independent readers for a reproducibility study. MMF demonstrated significantly lower values on average in the elderly cohort compared with the younger cohort for both ATT (decreased by $16.8 \%, \mathrm{p}=0.03$ ) and PTT (decreased by $23.0 \%, \mathrm{p}<0.01$ ). $\mathrm{T} 2_{\mathrm{MM}}$ and T1 did not show a significant nor a consistent difference between the young and elderly cohorts. For all MRI parameters, intraclass correlation coefficient (ICC) was higher than 0.98 , indicating excellent consistency between measurements performed by independent readers. MMF serving as a surrogate measure for collagen content, showed a significant decrease in elderly leg tendons. This study highlighted UTE-MRI-MT techniques as a useful quantitative method to assess the impact of aging on human tendons.

Tendons are responsible for transferring the mechanical loads generated by muscles to bones. Water may comprise over $60 \%$ of the total tendon weight ${ }^{1}$. Of the dry weight of the tendon, $60-85 \%$ is composed of collagen, particularly collagen type I, which is highly organized in the form of collagen fibrils, fibers, and bundles of fibers and fascicles ${ }^{1,2}$.

Magnetic resonance imaging (MRI), a non-invasive imaging modality, is routinely used for morphological evaluation of tendons ${ }^{3-5}$. Unfortunately, conventional clinical MRI sequences detect very low signal in tendons, presenting a significant challenge in the accurate assessment of tendons with clinical MRI ${ }^{6-8}$. Specifically, the detected signal intensity of a tissue in MRI depends on various factors, including transverse magnetization relaxation time $(\mathrm{T} 2)^{8,9}$. Tendons possess very short $\mathrm{T} 2$ relaxation times due to a high percentage of an organized 
collagenous matrix ${ }^{8}$. The low signal-to-noise ratio of tendons might limit differentiation of healthy tendon versus abnormal tissue.

Ultrashort echo time (UTE) MRI sequences are capable of detecting considerable signal of tendons before decay to background levels occurs ${ }^{8}$. Consequently, UTE-MRI has provided quantitative MRI-based assessments of tendons, such as apparent relaxation time of transverse magnetization (T2*), relaxation time of longitudinal magnetization (T1), and magnetization transfer (MT) measurements such as MT ratio (MTR $)^{6-8,10-16}$.

The other critical barrier to the use of clinical MRI and UTE-MRI for quantitative assessment of tendons is the confounding factor of the "magic angle effect." The magic angle effect is seen in collagen-rich tissues and is due to orientation sensitivity of dipolar interactions of proton nuclear spins $\mathrm{s}^{17}$. As the tissue fiber orientation approaches 55 degrees relative to the main magnetic field axis, $\mathrm{B}_{0}$, frequency changes from dipolar interactions are minimized, resulting in maximized signal intensity ${ }^{18}$. Earlier studies have reported that over $200 \%$ of change observed in MR properties of normal tendons and cartilage measured at 3T only resulted from differing orientations of the tissue relative to $\mathrm{B}_{0}{ }^{19-22}$.

Magnetization transfer (MT) modeling combined with UTE-MRI has recently been used to indirectly measure the macromolecular protons fraction (MMF) in different tissues ${ }^{20,21,23-28}$. Interestingly, two-pool UTE-MT modeling has demonstrated promise as a clinically compatible quantitative technique that is resistant to the magic angle effect ${ }^{20,21}$. Specifically, UTE-MT modeling has been recently implemented on cadaveric Achilles and rotator cuff tendons in order to assess MMF, and showed roughly no orientation angle sensitivity ${ }^{20,21}$. UTE-MT modeling relies on the phenomenon of magnetization transfer to quantify protons, including directly detected water protons (both free and restricted) and indirectly detected macromolecular protons, which possess $\mathrm{T} 2 *$ values too short for direct imaging, even with UTE sequences. This technique provides multiple parameters, including MMF, macromolecular relaxation time $\left(\mathrm{T} 2_{\mathrm{MM}}\right)$, and exchange rates ${ }^{20,21}$.

Despite the remarkably stable UTE-MT modeling results at different orientation angles, it is currently unclear how sensitive MT parameters are to tendons' compositional and structural changes through the aging process. If UTE-MT modeling demonstrates significant sensitivity to the aging process in tendon, it may be helpful in the detection of weakened tendon regions and in the prediction of potential tendon injuries.

The main objective of this study was to determine if UTE-MT modeling is a quantitative MRI technique sensitive to age-related changes in human leg tendons.

\section{Material and Methods}

Subject inclusion. A total of 26 healthy young female subjects ( $29 \pm 6$ years old) and 22 elderly female subjects ( $75 \pm 8$ years old) were recruited through posted flyers for leg MRI scans. The young healthy subjects were under 40 years old. Elderly female subjects were over 50 years old and in postmenopausal condition. Pregnant women and unhealthy volunteers were excluded after an initial screening questionnaire. No known tendon diseases were present in either cohort.

The institutional review board (IRB) of University of California, San Diego has approved this study. This study was conducted in accordance with applicable good clinical practice requirements and the relevant guidelines and regulations. Written informed consent was obtained from all patients.

UTE-MRI scanning protocol. The legs in all subjects were imaged using UTE-MRI sequences on a 3T MRI (MR750, GE Healthcare Technologies, WI, USA) using an eight-channel knee coil for both RF transmission and signal reception. Participants were asked to select their desirable leg to be scanned. The imaging slab was centered at the middle of the tibia and localized based on operator experience. To measure $\mathrm{T}_{1}$ as a prerequisite for the two-pool MT modeling, an actual flip angle-variable TR (AFI-VTR)-based 3D-UTE-Cones sequence (AFI: $\mathrm{TE}=0.032 \mathrm{~ms} ; \mathrm{TRs}=20 \mathrm{~ms}$ and $100 \mathrm{~ms} ; \mathrm{FA}=45^{\circ}$; VTR: TE $=0.032 \mathrm{~ms}$; TRs $=20,80$, and $150 \mathrm{~ms} ; \mathrm{FA}=45^{\circ}$; rectangular RF pulse with a duration of $150 \mu \mathrm{s}$ ) was performed with a total scan time of 20 minutes ${ }^{29}$. Additionally, a 3D-UTE-Cones-MT sequence (Fermi saturation pulse power $=500^{\circ}, 1000^{\circ}$, and $1500^{\circ}$; frequency offset $=2$, $5,10,20$, and $50 \mathrm{kHz} ; \mathrm{FA}=7^{\circ} ; 9$ spokes per MT preparation; rectangular RF excitation pulse of $100 \mu$ s) was performed for two-pool MT modeling with a total scan time of 14 minutes $^{30-32}$. Field of view (FOV), matrix dimension, nominal in-plane pixel size, and slice thickness were $14 \mathrm{~cm}, 160 \times 160,0.87 \mathrm{~mm}$, and $5 \mathrm{~mm}$, respectively.

MRI data analysis. Elastix software (open source software: http://elastix.isi.uu.nl/) was used to register all images to the first $\mathrm{T} 1$ image $(\mathrm{TR}=20 \mathrm{~ms})$ to compensate for potential subject motion. All scans were smoothed using a Gaussian filter with a $3 \times 3$ sub-window before T1 and MT measurements.

ROIs were selected by three experienced radiology trainees at the anterior tibialis tendon (ATT) and posterior tibias tendon (PTT). Figure 1 shows a schematic of the selected ROIs in a representative Cones UTE-MRI image from a 50 -year-old female subject $(\mathrm{TR}=20 \mathrm{~ms}$ and $\mathrm{TE}=2 \mathrm{~ms}$ ). For quality control purposes, the selected ATT and PTT ROIs by the trainees were overseen and validated by a board certified MSK radiologist for the first three datasets.

$\mathrm{T}_{1}$ was measured based on single-component exponential fittings $(S(T R) \propto 1-\exp (-T R / T 1)+$ constant $)$ on the acquired Cones-AFI-VTR data ${ }^{29}$. The acquired data with the set of MT saturation pulse powers $\left(500^{\circ}\right.$, $1000^{\circ}$, and $\left.1500^{\circ}\right)$ and frequency offsets $(2,5,10,20$, and $50 \mathrm{kHz})$ were fitted by a modified rectangular pulse approximation (mRP) approach which was previously described ${ }^{20,23,33}$. Super-Lorentzian line shape was used to model the macromolecular proton spectrum. The loss of the longitudinal magnetization of the macromolecular pool was also fitted by a Super-Lorentzian line shape function ${ }^{23}$.

The UTE-MT analysis was performed offline on the acquired DICOM images using an in-house code written in MATLAB (version 2016, Mathworks, Natick, MA, USA). A Levenberg-Marquardt algorithm was employed for the non-linear least-squares fitting in both UTE-MT modeling and T1 fitting within the earlier selected ROIs (Fig. 1). 


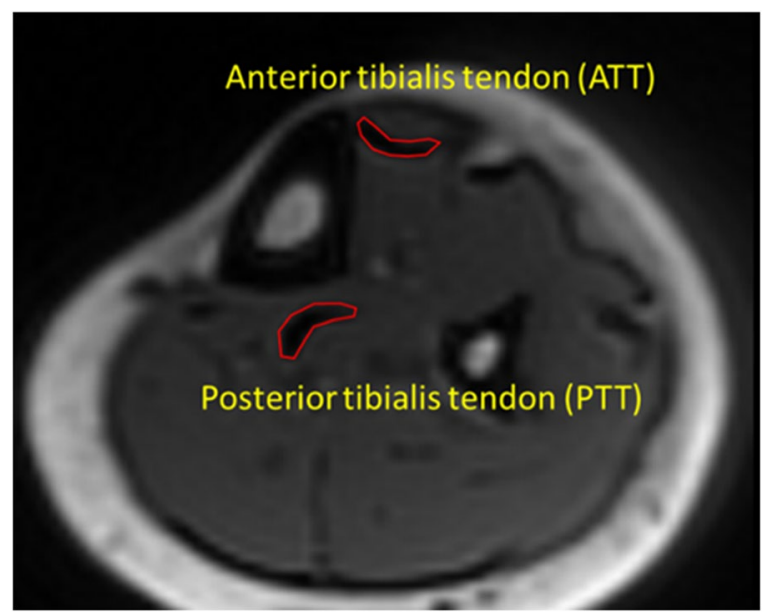

Figure 1. A representative Cones UTE-MRI image from a 50-year-old female subject (TR=20 ms and $\mathrm{TE}=2 \mathrm{~ms}$ ). Tibialis anterior and posterior tendons were obvious in the MRI images, and are indicated in red contours.

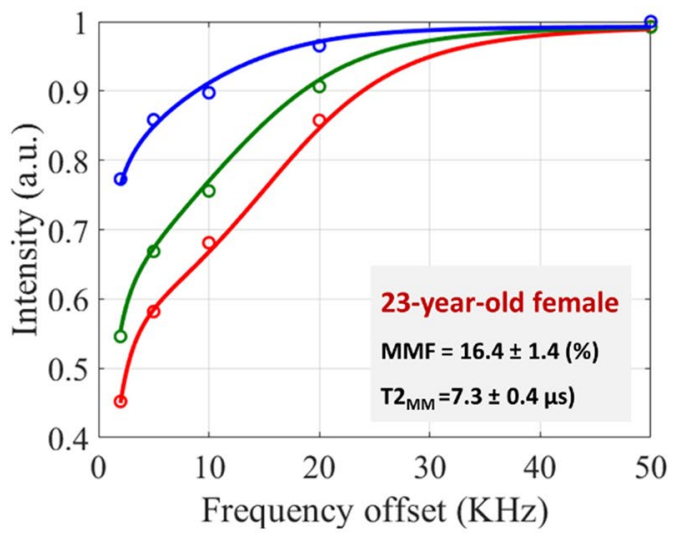

(a)

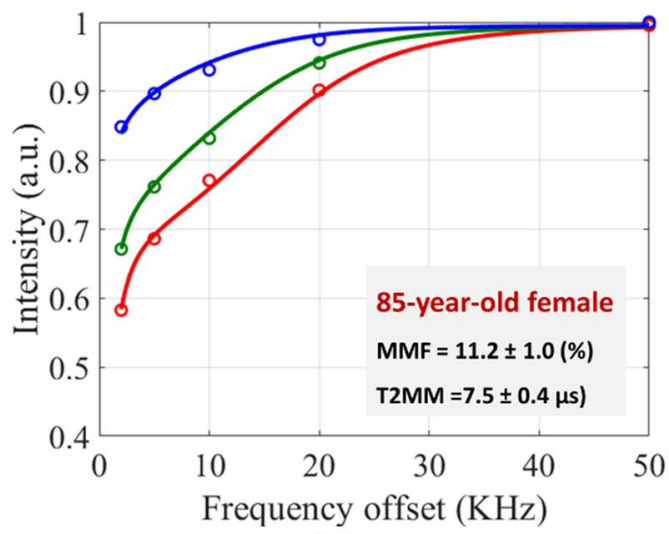

(b)

Figure 2. The two-pool MT modeling analyses in the anterior tibialis tendon (ATT) of (a) a young female subject and (b) an old female subject. Average saturated water signal in selected ROIs are depicted with circles for the three pulse saturation powers $\left(500^{\circ}\right.$ in blue, $1000^{\circ}$ in green, and $1500^{\circ}$ in red) and five frequency offsets $(5,10,20,50 \mathrm{kHz})$. Solid lines are fitting lines based on Super-Lorentzian shape functions. MMF and $\mathrm{T} 2_{\mathrm{MM}}$ refer to macromolecular fraction and macromolecular T2, respectively.

Statistical analysis. All statistical analyses were performed using MATLAB software. The differences in T1 and MT modeling results (MMF and $\mathrm{T} 2_{\mathrm{MM}}$ ) were compared between young and elderly cohorts using a two-sided Wilcoxon rank sum test, which is appropriate for unequal sample size ( $n=26$ for young cohort and $n=22$ for elderly cohort). P-values below 0.05 were considered significant. To investigate the reproducibility of the measurement process, intraclass correlation coefficient (ICC) was measured comparing the T1, MMF, and T2MM in all datasets as estimated by the three independent radiology trainees.

\section{Results}

Figure 2a,b show the two-pool MT modeling analyses within the defined ROIs of ATT tendon for a young female subject (23-year-old) and an old female subject (85-year-old), respectively. MT modeling was performed for five off-resonance frequencies and three MT saturation pulse power levels, including $500^{\circ}, 1000^{\circ}$, and $1500^{\circ}$, which are indicated with blue, green, and red lines. MMF was lower for older subjects in the presented examples.

Figure 3 shows the generated MMF maps for two young healthy (23 and 31-years-old) and two old (75 and 85 years old) female subjects. MMF appeared lower in older individuals compared with younger ones for the presented representative results.

Table 1 presents the estimated average and standard deviation values of T1, MMF, and $\mathrm{T}_{\mathrm{MM}}$ values in the ATT and PTT among young and old cohorts. Independent measurements by the three radiologists were averaged. ICC between the three independent measurements are also presented in Table 1 for T1, MMF, and T2 $2_{\mathrm{MM}}$ values. For all MRI parameters, ICC was higher than 0.98, which indicates excellent consistency between measurements 


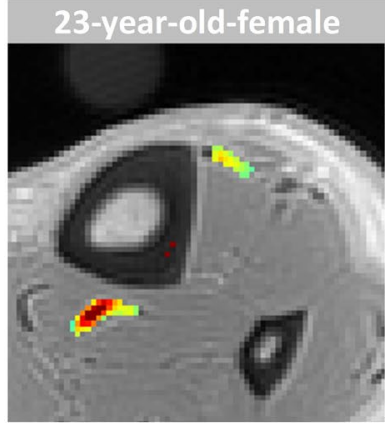

(a)

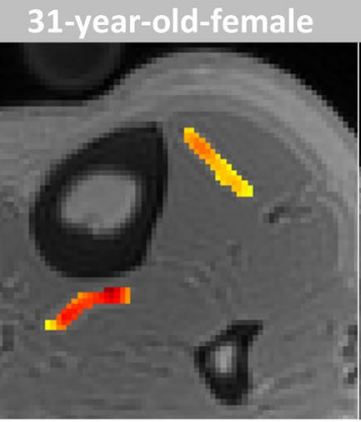

(b)

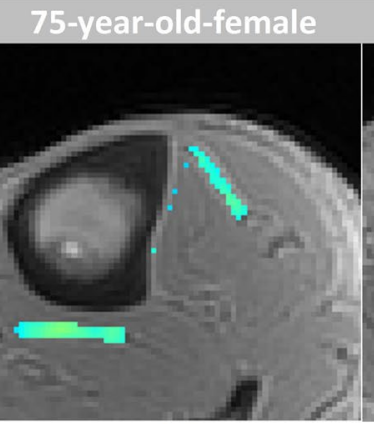

(c)

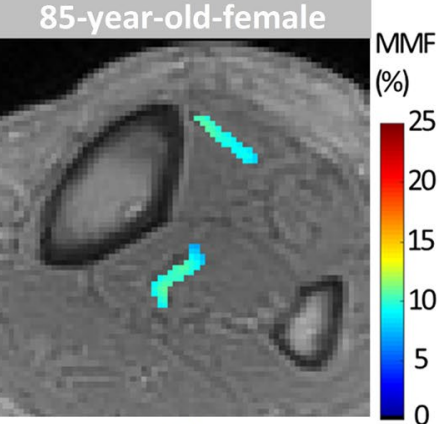

(d)

Figure 3. Generated macromolecular proton fraction (MMF) maps for two young healthy subjects, (a) 23-yearold female and (b) 31-year-old female, and two old subjects, (c) 75-year-old female and (d) 85-year-old female.

\begin{tabular}{|l|l|c|c|c|}
\hline & & T1 $(\mathbf{m s})$ & MMF $(\%)$ & T2 \\
\hline \multirow{3}{*}{ young } & $(\boldsymbol{\mu s})$ \\
\cline { 2 - 5 } & ATT & $823 \pm 156$ & $17.0 \pm 5.0$ & $7.3 \pm 0.2$ \\
\cline { 2 - 5 } elderly & PTT & $735 \pm 86$ & $21.3 \pm 4.8$ & $7.3 \pm 0.2$ \\
\cline { 2 - 5 } & ATT & $789 \pm 154$ & $14.1 \pm 3.9$ & $7.3 \pm 0.3$ \\
\hline \multirow{2}{*}{ ICC } & & $762 \pm 123$ & $16.4 \pm 3.3$ & $7.1 \pm 0.3$ \\
\hline
\end{tabular}

Table 1. Mean and standard deviation of T1 and UTE-MT measurements in anterior and posterior tibialis tendons among young and old cohorts. The intraclass correlation coefficients (ICC) were also measured between the three independent readers.

\begin{tabular}{|c|c|c|c|}
\hline & $\mathrm{T} 1$ & MMF & $\mathrm{T} \mathbf{2}_{\mathrm{MM}}$ \\
\hline ATT & $\begin{array}{l}-4.2 \% \\
(\mathrm{p}=0.51)\end{array}$ & $\begin{array}{l}-16.8 \% \\
(p=0.03)\end{array}$ & $\begin{array}{l}-0.5 \% \\
(p=0.56)\end{array}$ \\
\hline PTT & $\begin{array}{l}3.6 \% \\
(p=0.67)\end{array}$ & $\begin{array}{l}-23.0 \% \\
(p<0.01)\end{array}$ & $\begin{array}{l}-2.0 \% \\
(\mathrm{p}=0.02)\end{array}$ \\
\hline
\end{tabular}

Table 2. Average percentage differences as well as Wilcoxon rank sum test results in $\mathrm{T} 1, \mathrm{MMF}$, and $\mathrm{T} 2_{\mathrm{MM}}$ of ATT and PTT tendons between elderly and young cohorts.

performed by independent readers. On average, MMF showed lower values for both ATT and PTT in the elderly cohort compared with young cohort.

Average percentage differences of UTE-MRI measures in ATT and PTT tendons between elderly and young cohorts, as well as Wilcoxon rank sum test results, are presented in Table 2. MMF demonstrated significantly lower values on average in the elderly cohort compared with the younger cohort at both ATT $(-16.8 \%, p=0.03)$ and PTT $(-23.0 \%, \mathrm{p}<0.01)$ tendons.

Figures $4 \mathrm{a}$,b show barplots of average MMF values in the ATT and PTT of young and elderly cohorts. As seen in Tables 1 and 2 and in Figure 4, the MMF difference between young and elderly cohorts was greater in the PTT compared with ATT.

\section{Discussion}

UTE-MT modeling acquired with the 3D-UTE-Cones sequence was employed to estimate the age-related differences of collagen proton fraction in human tibial tendons. Aging in general affects the quality, composition, and performance of the tendons ${ }^{34-37}$. UTE-MT modeling as an orientation-insensitive MRI technique and a noninvasive tool to detect collagen fraction changes throughout aging may potentially help in the detection of weakened tendons and in prediction of potential tears or injuries. Timely detection of collagen fraction variation in tendons becomes more crucial due to the age-dependent reduction of tendon's cellular activities that may impair the tissue repair process.

Aging can affect tendons' biomechanics directly and lead to weaker tendons as a result of biological changes $^{34,35,37}$. Tendons' stiffness reduction through aging has been reported from $2 \%$ up to $55 \%$ depending on the studied tendons ${ }^{35,36}$. Tendons may also adapt their microstructure and composition as a result of decreasing mechanical loads due to weakening muscles and lowering levels of physical activity ${ }^{35,37}$. Age-related changes in muscle strength measuring up to $52 \%$ have been reported in the literature ${ }^{35}$. Age-related changes in the cross-section area of tendon have been also investigated, though the variation patterns between different studies were controversial ${ }^{35,36,38}$. 


\section{Anterior tibialis tendon (ATT)}

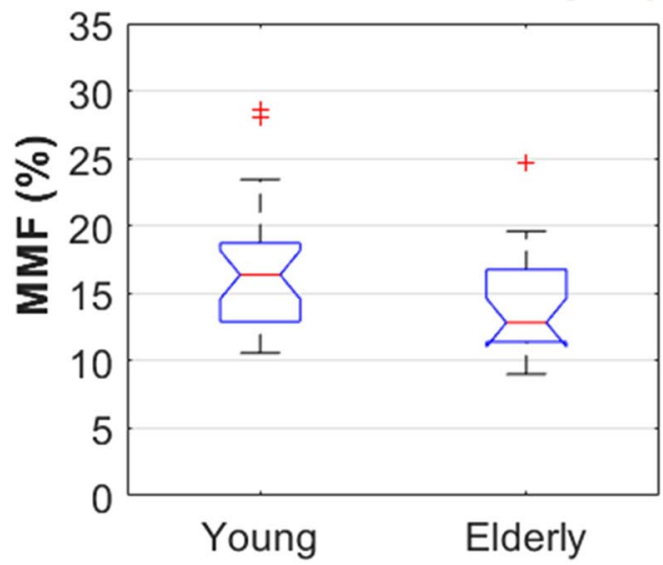

(a)

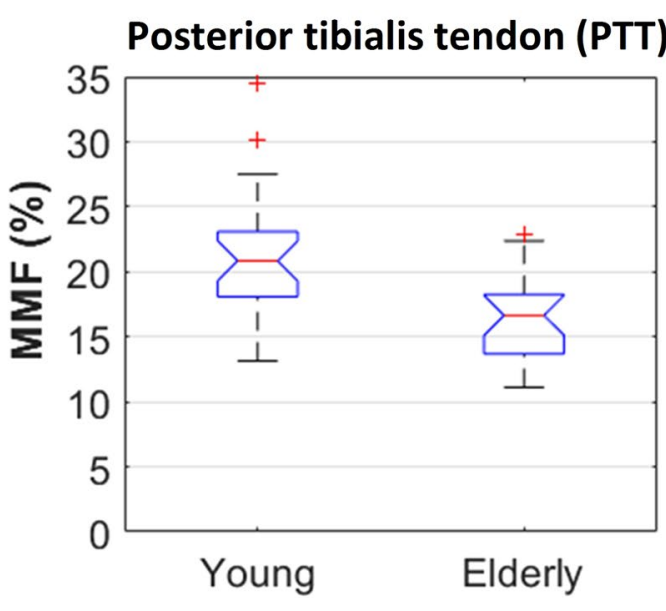

(b)

Figure 4. Average MMF in anterior and posterior tibialis tendons for young and elderly cohorts. Average MMF values were significantly lower in tendons of elderly cohort $(\mathrm{p}<0.01)$. The central mark in boxplots indicates the median, while the bottom and top edges of the boxes indicate the 25th and 75th percentiles, respectively. ' + ' symbol refers to outliers. The elderly cohort showed significantly lower average MMF. The MMF difference between young and elderly cohorts was greater in PTT tendon.

The MMF of both tendons studied here (anterior and posterior tibialis tendons) were significantly lower in the elderly cohort compared with the young cohort (Tables 1 and 2, Fig. 4). MMF reduction most likely indicates a reduction in the collagen content in the studied tendons, although increases in water content can also contribute. The MMF difference in the posterior tibialis tendon was greater compared with the anterior tendon. The PTT plays a key role as a dynamic supporter of the longitudinal arch of the foot, and thusly be affected more intensively by lower activity level in older subjects due to this larger role in physical activity. In a random sample of 1,000 women over 40 years of age, 3.3\% had undiagnosed PTT dysfunction ${ }^{39}$. The collagen fibers quality in tendons might not be affected significantly by aging, therefore $\mathrm{T} 1$ and $\mathrm{T} 2_{\mathrm{MM}}$ did not show consistent and significant differences in both studied tendons between young and elderly cohorts. Decreases in such relaxation times can indicate a less viscoelastic collagen fiber.

Hodgson et al. ${ }^{40}$ used a 2D UTE version of MT modeling and found lower MMF values in the Achilles tendon of a patient with psoriatic arthritis (MMF 16\%) compared with healthy volunteers (MMF 21\%). Ma et al. previously used the 2D UTE-MT technique on Achilles tendon specimens and demonstrated the orientation-insensitivity of the UTE-MT modeling, with mean MMF values of $20 \%{ }^{20}$. Zhu et al. later used 3D Cones UTE-MT modeling to assess rotator cuff tendon specimens and reported significantly lower MMF values for specimens with severe tendinopathy compared with specimens with mild tendinopathy (16\% vs. $12 \%$, respectively $)^{21}$. The values of ATT and PTT MMF in our study are in range with these previously reported values ${ }^{20,21}$. However, it is important to note that tendon composition, collagen morphology, and biomechanical properties vary not only between tendons, but in a region-dependent manner within the same tendon ${ }^{7,13,41}$. In young and elderly mice, Wood et al. found a substantially increased tangent modulus in the proximal region of the ATT (near the muscle) compared with the rest of the tendon more distally, although all regions of tendons were stiffer with advancing age ${ }^{41}$.

Recently, the 3D Cones UTE-MT modeling has been employed to evaluate MMF in cortical bone organic matrix and to investigate MMF correlations with bone's microstructural and mechanical properties ${ }^{24-28}$. MMF values reported in cortical bone ( $40-70 \%)$ have been much higher than tendon due to the much smaller water fraction ( $\sim 20 \%$ bone vs $\sim 60-70 \%$ tendon) and corresponding higher fraction of macromolecules.

The first limitation of this study was the relatively long scan time (approx. 34 mins), which may have made it difficult for subjects to remain motionless during the scan. Although, motion was not substantial on the images obtained from most of our subjects, image registration could help the cases where motion occurred between series. Employing different accelerating techniques, such as stretching the readout trajectory, could be used to accelerate the technique and to limit the scans to 20 minutes with negligible resultant errors ${ }^{42}$. Secondly, the fat present in tendon was not taken into account for this study. Intratendinous fat would not be expected to be substantial in the location we imaged, although it may be greater near the enthesis ${ }^{43}$. The potential impacts of fat chemical shift on the estimated MMF values in tendons need to be studied in a future investigation. Reducing the fat signal contamination can be achieved using different fat suppression techniques ${ }^{44,45}$. Thirdly, the presented technique was translated to the leg tendons in healthy young and elderly subjects with no known clinical tendon diseases. However, we cannot exclude subclinical tendinopathy in some cases. Furthermore, our screening questionnaire did not specifically inquire about recent drug therapies. Reversible changes in tendons after ciprofloxacin administration were shown by Juras et al. ${ }^{46}$ using both sodium MRI and glycosaminoglycan chemical exchange saturation transfer at 7T. Although special hardware is required for sodium and 7T MRI, future investigations assessing the sensitivity between all of these MRI techniques in different disease conditions 
should be performed. Fourthly, this study only included female participants that was meant to avoid misinterpretation caused by gender-related differences. According to the literature, female's tendon structure is different from male's tendon structure ${ }^{47}$. Moreover, female age-related tendon variation can be different from variations in male cohorts ${ }^{48}$. Nevertheless, performing a similar study on male cohorts and comparing the results with the presented findings on female cohorts will be a future step. This future study will also examine the robustness of the presented sequences and MMF measures on the male cohorts. Fifthly, this investigation only included tibial tendons which their pathology is quite common, in particular involving the $\mathrm{PTT}^{39,49,50}$. However other tendons such as patellar and Achilles tendons are also of clinical significance that can be evaluated using the presented MRI techniques in a future well-designed study.

\section{Conclusion}

Two-pool UTE-MT modeling was investigated for its capability to assess age-related collagen fraction differences in human leg tendons. MMF obtained from MT modeling, as an index for collagen fraction in tendons, showed significantly lower $(\sim 20 \%)$ values in anterior and posterior tibialis tendons of elderly subjects compared with young subjects. This study highlighted UTE-MT MRI techniques as useful methods to assess tendons in the aging population, which may be used in the future for detection of weakened tendons and prediction of potential injuries.

Received: 8 July 2019; Accepted: 11 November 2019;

Published online: 29 November 2019

\section{References}

1. Aparecida de Aro, A., de Campos Vidal, B. \& Pimentel, E. R. Biochemical and anisotropical properties of tendons. Micron 43, 205-214 (2012).

2. Khan, K. M., Cook, J. L., Bonar, F., Harcourt, P. \& Astrom, M. Histopathology of common tendinopathies: update and implications for clinical management. Sport. Med. 27, 393-408 (1999).

3. Pierre-Jerome, C., Moncayo, V. \& Terk, M. R. MRI of the Achilles tendon: a comprehensive review of the anatomy, biomechanics, and imaging of overuse tendinopathies. Acta radiol. 51, 438-454 (2010).

4. Weinreb, J. H. et al. Tendon structure, disease, and imaging. Muscles. Ligaments Tendons J. 4, 66-73 (2014).

5. Hodgson, R., O’Connor, P. J. \& Grainger, A. J. Tendon and ligament imaging. Br. J. Radiol. 85, 1157-1172 (2012).

6. Du, J.et al. Orientational analysis of the Achilles tendon and enthesis using an ultrashort echo time spectroscopic imaging sequence. Magn. Reson. Imaging 28, 178-184 (2010).

7. Juras, V. et al. Regional variations of T2* in healthy and pathologic achilles tendon in vivo at 7 Tesla: Preliminary results. Magn. Reson. Med. 68, 1607-1613 (2012).

8. Chang, E. Y., Du, J. \& Chung, C. B. UTE imaging in the musculoskeletal system. J. Magn. Reson. Imaging 41, 870-883 (2015).

9. Hendee, W. R. \& Morgan, C. J. Magnetic resonance imaging. Part I-physical principles. West. J. Med. 141, 491-500 (1984).

10. Syha, R. et al. Short-term exercise-induced changes in hydration state of healthy achilles tendons can be visualized by effects of offresonant radiofrequency saturation in a three-dimensional ultrashort echo time MRI sequence applied at 3 Tesla. J. Magn. Reson. Imaging 40, 1400-1407 (2014).

11. Du, J. et al. Qualitative and quantitative ultrashort echo time (UTE) imaging of cortical bone. J. Magn. Reson. 207, 304-311 (2010).

12. Koff, M. F., Pownder, S. L., Shah, P. H., Yang, L. W. \& Potter, H. G. Ultrashort echo imaging of cyclically loaded rabbit patellar tendon. J. Biomech. 47, 3428-3432 (2014).

13. Juras, V. et al. Bi-exponential T2*analysis of healthy and diseased Achilles tendons: An in vivo preliminary magnetic resonance study and correlation with clinical score. Eur. Radiol. 23, 2814-2822 (2013).

14. Chang, E. Y., Du, J., Bae, W. C., Statum, S. \& Chung, C. B. Effects of Achilles tendon immersion in saline and perfluorochemicals on $\mathrm{T} 2$ and T2*. J. Magn. Reson. Imaging 40, 496-500 (2014).

15. Chang, E. Y., Bae, W. C., Statum, S., Du, J. \& Chung, C. B. Effects of repetitive freeze-thawing cycles on T2 and T2* of the Achilles tendon. Eur. J. Radiol. 83, 349-353 (2014).

16. Du, J.et al. Ultrashort TE T1rho (UTE T1rho) imaging of the Achilles tendon and meniscus. Magn. Reson. Med. 64, 834-842 (2010).

17. Berendsen, H. J. C. Nuclear Magnetic Resonance Study of Collagen Hydration. J. Chem. Phys. 36, 3297-3305 (1962).

18. Krasnosselskaia, L. V., Fullerton, G. D., Dodd, S. J. \& Cameron, I. L. Water in tendon: Orientational analysis of the free induction decay. Magn. Reson. Med. 54, 280-288 (2005).

19. Chang, E. Y., Szeverenyi, N. M., Statum, S. \& Chung, C. B. Rotator cuff tendon ultrastructure assessment with reduced-orientation dipolar anisotropy fiber imaging. Am. J. Roentgenol. 202, 376-378 (2014).

20. Ma, Y., Shao, H., Du, J. \& Chang, E. Y. Ultrashort echo time magnetization transfer (UTE-MT) imaging and modeling: magic angle independent biomarkers of tissue properties. NMR Biomed. 29, 1546-1552 (2016).

21. Zhu, Y. et al. Rotator cuff tendon assessment using magic-angle insensitive 3D ultrashort echo time cones magnetization transfer (UTE-Cones-MT) imaging and modeling with histological correlation. J. Magn. Reson. Imaging 48, 160-168 (2018).

22. Shao, H. et al. Magic angle effect plays a major role in both T1rho and T2 relaxation in articular cartilage. Osteoarthr. Cartil. 25, 2022-2030 (2017).

23. Ma, Y., Chang, E. Y., Carl, M. \& Du, J. Quantitative magnetization transfer ultrashort echo time imaging using a time-efficient 3D multispoke Cones sequence. Magn. Reson. Med. 00, 1-9 (2017).

24. Jerban, S. et al. Volumetric Mapping of Bound and Pore Water as well as Collagen Protons in Cortical Bone Using 3D Ultrashort Echo Time Cones MR Imaging Techniques. Bone 127, 120-128 (2019).

25. Jerban, S. et al. Collagen proton fraction from ultrashort echo time magnetization transfer (UTE-MT) MRI modelling correlates significantly with cortical bone porosity measured with micro-computed tomography ( $\mu \mathrm{CT}$ ). NMR Biomed. 32, 1-10 (2019).

26. Jerban, S. et al. Ultrashort echo time magnetic resonance imaging (UTE-MRI) of cortical bone correlates well with histomorphometric assessment of bone microstructure. Bone 123, 8-17 (2019).

27. Jerban, S. et al. Detecting stress injury (fatigue fracture) in fibular cortical bone using quantitative ultrashort echo timemagnetization transfer (UTE-MT): An ex vivo study. NMR Biomed. 31, e3994 (2018).

28. Jerban, S. et al. Assessing cortical bone mechanical properties using collagen proton fraction from ultrashort echo time magnetization transfer (UTE-MT) MRI modeling. Bone Reports 8 (2019).

29. Ma, Y. et al. Accurate T 1 mapping of short T 2 tissues using a three-dimensional ultrashort echo time cones actual flip angle imaging-variable repetition time (3D UTE-Cones AFI-VTR) method. Magn. Reson. Med. 00, 1-11 (2018).

30. Gurney, P. T., Hargreaves, B. A. \& Nishimura, D. G. Design and analysis of a practical 3D cones trajectory. Magn. Reson. Med. 55, $575-582(2006)$. 
31. Carl, M., Bydder, G. M. \& Du, J. UTE imaging with simultaneous water and fat signal suppression using a time-efficient multispoke inversion recovery pulse sequence. Magn. Reson. Med. 76, 577-582 (2015).

32. Ma, Y. et al. Short T 2 imaging using a 3D double adiabatic inversion recovery prepared ultrashort echo time cones (3D DIR-UTECones) sequence. Magn. Reson. Med. 00, 1-9 (2017).

33. Ma, Y., Tadros, A., Du, J. \& Chang, E. Y. Quantitative two-dimensional ultrashort echo time magnetization transfer (2D UTE-MT) imaging of cortical bone. Magnetic Resonance in Medicine, https://doi.org/10.1002/mrm.26846 (2017).

34. Kannus, P., Paavola, M. \& Józsa, L. Aging and Degeneration of tendons. In Tendon Injuries 25-31, https://doi.org/10.1007/b137778 (2005).

35. McCrum, C. et al. Alterations in leg extensor muscle-tendon unit biomechanical properties with ageing and mechanical loading. Front. Physiol. 9, 1-7 (2018).

36. Carroll, C. C. et al. Influence of aging on the in vivo properties of human patellar tendon. J. Appl. Physiol. 105, 1907-1915 (2008).

37. Svensson, R. B., Heinemeier, K. M., Couppé, C., Kjaer, M. \& Magnusson, S. P. Effect of aging and exercise on the tendon. J. Appl. Physiol. 121, 1353-1362 (2016).

38. Magnusson, S. P. et al. Increased cross-sectional area and reduced tensile stress of the Achilles tendon in elderly compared with young women. J. Gerontol. A. Biol. Sci. Med. Sci. 58, 123-7 (2003).

39. Kohls-Gatzoulis, J., Woods, B., Angel, J. C. \& Singh, D. The prevalence of symptomatic posterior tibialis tendon dysfunction in women over the age of 40 in England. Foot Ankle Surg. 15, 75-81 (2009).

40. Hodgson, R. J. et al. Quantitative magnetization transfer ultrashort echo time imaging of the Achilles tendon. Magn. Reson. Med. 65, $1372-1376(2011)$.

41. Wood, L. K., Arruda, E. M. \& Brooks, S. V. Regional stiffening with aging in tibialis anterior tendons of mice occurs independent of changes in collagen fibril morphology. J. Appl. Physiol. 111, 999-1006 (2011).

42. Wan, L. et al. Fast quantitative three-dimensional ultrashort echo time (UTE) magnetic resonance imaging of cortical bone using extended cones sampling. Magn. Reson. Med., https://doi.org/10.1002/mrm.27715 (2019).

43. Benjamin, M. et al. Adipose tissue at entheses: The rheumatological implications of its distribution. A potential site of pain and stress dissipation? Ann. Rheum. Dis. 63, 1549-1555 (2004).

44. Gee, C. S. et al. Validation of bone marrow fat quantification in the presence of trabecular bone using MRI. J. Magn. Reson. Imaging 42, 539-44 (2015).

45. Jang, H. et al. Fat Suppression for Ultrashort Echo Time Imaging Using a Single Point Dixon Method. NMR Biomed. e4069 (2019).

46. Juras, V. et al. Multiparametric MR Imaging Depicts Glycosaminoglycan Change in the Achilles Tendon during Ciprofloxacin Administration in Healthy Men: Initial Observation. Radiology 275, 763-771 (2015).

47. Sarver, D. C. et al. Sex differences in tendon structure and function. J. Orthop. Res. 35, 2117-2126 (2017).

48. Pease, L. I. et al. Cross platform analysis of transcriptomic data identifies ageing has distinct and opposite effects on tendon in males and females. Sci. Rep. 7, 1-20 (2017).

49. Carmody, D., Bubra, P., Keighley, G. \& Rateesh, S. Posterior tibial tendon dysfunction: An overlooked cause of foot deformity. J. Fam. Med. Prim. Care 4, 26 (2015).

50. Kohls-Gatzoulis, J., Angel, J. \& Singh, D. Tibialis posterior dysfunction as a cause of flatfeet in elderly patients. Foot 14, 207-209 (2004).

\section{Acknowledgements}

The authors acknowledge grant support from NIH (R21AR073496, R01AR075825, 1R01NS092650, R01AR062581-06) and VA Clinical Science and Rehabilitation R\&D Awards (I01CX001388 and I01RX002604).

\section{Author contributions}

Study design: S.J., J.D. and E.Y.C. Study conduct: S.J., Y.M. and N.L. MRI sequence development: Y.M. In vivo MRI scans: S.J. and Y.M. MRI Data registration: Z.W., M.W. and Z.C.S. MRI data analysis and interpretation: S.J., B.N., A.A., H.S. Drafting manuscript: S.J., N.L. and E.Y.C. Revising and approving manuscript content: S.J., Y.M., B.N., A.A., H.S., Z.W., N.L., M.W., Z.C., J.D. and E.Y.C. E.Y.C. and S.J. take responsibility for the integrity of the data analysis.

\section{Competing interests}

The authors declare no competing interests.

\section{Additional information}

Correspondence and requests for materials should be addressed to S.J. or E.Y.C.

Reprints and permissions information is available at www.nature.com/reprints.

Publisher's note Springer Nature remains neutral with regard to jurisdictional claims in published maps and institutional affiliations.

(c) (i) Open Access This article is licensed under a Creative Commons Attribution 4.0 International

License, which permits use, sharing, adaptation, distribution and reproduction in any medium or format, as long as you give appropriate credit to the original author(s) and the source, provide a link to the Creative Commons license, and indicate if changes were made. The images or other third party material in this article are included in the article's Creative Commons license, unless indicated otherwise in a credit line to the material. If material is not included in the article's Creative Commons license and your intended use is not permitted by statutory regulation or exceeds the permitted use, you will need to obtain permission directly from the copyright holder. To view a copy of this license, visit http://creativecommons.org/licenses/by/4.0/.

(c) The Author(s) 2019 